\title{
Deposition of Tungsten Thin Films by Magnetron Sputtering for Large-Scale Production of Tungsten-Based Transition-Edge Sensors
}

\author{
A. H. Abdelhameed ${ }^{1}$ (D) . G. Angloher ${ }^{1} \cdot$ P. Bauer $^{1} \cdot$ A. Bento B $^{1,3} \cdot$ E. Bertoldo $^{1}$. \\ L. Canonica ${ }^{1}$ - D. Fuchs ${ }^{1}$ - D. Hauff ${ }^{1}$. N. Ferreiro lachellini ${ }^{1} \cdot$ M. Mancuso ${ }^{1}$. \\ F. Petricca ${ }^{1} \cdot$ F. Pröbst ${ }^{1} \cdot$ J. Riesch ${ }^{2} \cdot$ J. Rothe $^{1}$
}

Received: 19 August 2019 / Accepted: 17 January 2020 / Published online: 1 February 2020

(c) The Author(s) 2020

\begin{abstract}
To cope with the foreseen demand for tungsten-based TESs in the current and future phases of the CRESST experiment, we investigated the possibility to implement a reliable, simple and reproducible fabrication method using sputtering. In this contribution, we present the method under development for tungsten deposition using conventional magnetron sputtering with xenon as sputtering gas. TESs with transition temperatures $\left(T_{c}\right)$ down to $15 \mathrm{mK}$ have been obtained with transition widths smaller than $1 \mathrm{mK}$. We also give a first assessment on the reproducibility of the process and present the potential for tuning of the $T_{c}$.
\end{abstract}

Keywords TES $\cdot$ Tungsten $\cdot$ Sputtering $\cdot$ Xenon $\cdot$ Dark matter $\cdot$ CRESST

\section{Introduction}

Superconducting transition-edge sensors (TESs) are used as ultrasensitive thermometers to measure temperature changes in the $\mu K$ range [1] and have attracted quite some attention in the last few decades due to their sensitivity. Such sensors are widely adopted in low-temperature detectors and are used in many different applications, e.g., particle physics, astroparticle physics and astronomy.

A. H. Abdelhameed aab@mpp.mpg.de

1 Max-Planck-Institut für Physik, 80805 Munich, Germany

2 Max-Planck-Institut für Plasmaphysik, 85748 Garching, Germany

3 Departamento de Fisica, Universidade de Coimbra, 3004516 Coimbra, Portugal 


\subsection{TESs in CRESST Experiment}

In the framework of the CRESST direct dark matter search [2, 3] (Cryogenic Rare Events Search with Superconducting Thermometers), tungsten TESs are used as sensing elements. Detectors in CRESST consist of a target crystal which has a mass of a few tens of grams, combined with a TES. These detectors are operated as cryogenic calorimeters at $\sim 10 \mathrm{mK}$. The main detection channel is scattering of hypothetical dark matter particles off the target crystal nuclei. The deposited energy leads to a measurable temperature rise in the temperature sensor. We use tungsten-based TESs since tungsten has a $T_{c} \sim 15.4 \mathrm{mK}$ (in the bulk form) which is the closest to our design goal. The measured temperature shift of the sensor, $\Delta T$, is directly proportional to the energy deposition detected by the sensor, $\Delta E$, and inversely proportional to the heat capacity of the sensor, $c$, where $\Delta T=\frac{\Delta E}{c}$. At this low temperature, tungsten has a relatively low heat capacity of $c[\mathrm{~J} /(\mathrm{g} \mathrm{K})] \approx 7.94 \times 10^{-6} \times T$ ensuring a large temperature response [4]. However, thin films usually behave differently as they do in the bulk form; the transition temperature, $T_{c}$, the width of the transition, $\Delta T c$, and the shape of the transition are strongly dependent on the film quality and can differ from what is commonly manifested by the bulk form [5].

Previously, we have produced tungsten films in an optimized e-beam evaporation system. However, evaporation is a time-consuming process (one week/run), involves high temperatures $\left(\sim 600^{\circ} \mathrm{C}\right)$, has low throughput (given the current size of CRESST-III detectors and the standard production procedure, i.e., sensors directly evaporated on the absorber crystals, up to four detectors can be produced in a single evaporation run) and has low yield. For the next CRESST upgrade, a few hundreds of detectors are foreseen. This number of detectors will require more production capacity than that of our evaporation machine. A promising candidate to substitute evaporation is sputtering.

\subsection{Superconducting Tungsten Thin Films}

The transition temperature and the transition shape (steepness) are two parameters that determine the performance of the detector. There are many aspects of the thin film deposition process that may affect the thin film composition and its crystalline quality and, as a consequence, the transition behavior. Besides these, there might be effects related to the final geometry of the sensors that should not be neglected, but unfortunately, a full understanding of what governs the $T_{c}$ in thin films has not yet been reached. Some parameters that may affect the $T_{c}$ are discussed in more detail in the following sections.

\subsubsection{Impurities}

The presence of impurities in tungsten films is known to strongly alter the $T_{c}$ or completely suppress the transition. Contamination of films can originate from the growth process itself. During film growth, any volatile contaminant or gas impurity 
can be easily incorporated in the film. In addition, the substrates may carry organic or inorganic residuals or water from the cleaning process. Even in highly controlled environments, tungsten may react with substrates at high temperatures, or even at room temperature. Some common tungsten compounds have shown superconductivity at relatively higher temperatures. $\beta$-W is known to be an unstable phase and to exist, sometimes, in grown tungsten films [6]. It also shows a wide range of $T_{c}$ up to $4 \mathrm{~K}$ [7]. Impurities play a big role in stabilizing that phase [7]. Less pure $\mathrm{W}$ films, or films where a high Tc is observed, are likely to be called $\beta$-W due to the technical difficulty of characterizing amorphous or nanocrystalline films, i.e., accurately quantifying whether the impurities form a stoichiometric phase with tungsten, or exist somewhere else, e.g., in the grain boundaries. Among impurities that cause high $T_{c}$ with respect to the nominal value, we can mention $\mathrm{W}$-silicide $\left(T_{c}<2.84 \mathrm{~K}\right.$ ) [8], W-carbide $\left(T_{c}<10 \mathrm{~K}\right)$ [9], W-nitride $\left(T_{c}<4.85 \mathrm{~K}\right)$ [10], W-oxide $\left(T_{c}<132 \mathrm{~K}\right.$ ) [11]. In contrast to these impurities, ferromagnetic impurities shift the $T_{c}$ to lower temperatures or completely suppress the transition [12].

\subsubsection{Argon Retention}

Retention of argon atoms in tungsten films cannot be easily avoided in sputtered films in Ar atmosphere. The Ar amount usually retained in sputtered films is $\sim 1-2 \%$, depending on the deposition parameters [13]. Such an unavoidable impurity may deteriorate the film quality in terms of superconductivity even without a chemical reaction with $\mathrm{W}$. As a matter of fact, it has been shown that noble gas impurities like He may stimulate superconductivity in naturally not superconducting elements, e.g., palladium thin films when irradiated with low energetic $\mathrm{He}^{+}$ions [14].

\subsubsection{Stress}

Stress is known to shift the $T_{c}$ of superconductors (e.g., see [15]). Residual stress is an intrinsic feature of grown thin films and can be either tensile or compressive. It arises from the fact that, as the film grows, a columnar microstructure dominates. The competition between the growing grains, the bombardment from the reflected neutral atoms, or the lattice parameter mismatch between the substrate and the film may lead to a stressed film (for more details, see [13]). Another type of stress is thermal expansion coefficient mismatch between films and substrates. Since the film will be operated at cryogenic temperatures, the difference in contraction between the film and the substrate will be relatively large which introduces stress in the film. The linear thermal expansion coefficient of tungsten is smaller than almost all typically used substrates and bigger than that of $\mathrm{Si}[16,17]$; hence, it is not possible to avoid such mismatch.

Our approach to overcome this obstacle is to engineer our films to have a residual amount of stress after deposition that will cancel out the induced stress due to the thermal expansion mismatch at low temperatures. Such engineering is possible by sputtering. 


\subsubsection{Xenon as a Sputtering Gas}

Mamor et al. [18] have shown a comparison of the residual stress in films produced with Ar versus Xe as a function of sputtering gas pressure. Based on their results, one can infer that a stress-free film can be deposited at a pressure around $1 \mathrm{~Pa}$ for $\mathrm{Xe}$ compared with $7 \mathrm{~Pa}$ for Ar. The relatively high pressure for Ar may result in a higher Ar concentration in the film, which we would like to avoid. On the other hand, Xe is less likely to be trapped both due to its atomic size and to the relatively low sputtering pressure.

\section{Experimental Methods}

Tungsten films $\sim 200 \mathrm{~nm}$ thick were deposited on single crystal Si(100) substrates with $\sim 30 \mathrm{~nm}$ thermally grown $\mathrm{SiO}_{2}$. We used a commercial sputtering system at Max Planck Institute for Plasma Physics in Garching. Substrates were diced from a 6 -inch wafer into $(10 \times 20) \mathrm{mm}^{2}$. The samples were degreased using acetone in an ultrasonic bath for $5 \mathrm{~min}$ followed by IPA for additional $5 \mathrm{~min}$ and then were dried with nitrogen gas. The system has two cathodes in a confocal configuration with a $25^{\circ}$ inclination to the substrate holder and a center-to-center distance $\sim 10 \mathrm{~cm}$. A 3 -in. tungsten target of $99.95 \%$ purity (Lesker) was used. The base pressure before deposition was $\sim 2.0 \times 10^{-6} \mathrm{~Pa}$. The chamber pressure during deposition was varied between 0.6 and $2.5 \mathrm{~Pa}$ for different runs in steps of $0.1 \mathrm{~Pa}$ while keeping all other parameters constant to study the effect of Xe pressure on $T_{c}$. Xe gas with purity of $5 N .3$ was admitted to the chamber using a mass flow controller (MFC) with flow between 9 and $25 \mathrm{sccm}$ to adjust the corresponding pressure. A DC power of $300 \mathrm{~W}$ was applied on the magnetron during all runs. Prior to the deposition, the samples were plasma cleaned using $50 \mathrm{~W}$ RF bias for $5 \mathrm{~min}$. While the magnetron shutter was closed, the target was sputtered clean for 5 min using the same power as during deposition. The deposition time was $5 \mathrm{~min}$ for all runs. To accurately study the effect of one parameter (pressure) while keeping all other parameters constant and without breaking the vacuum, a sample holder that can be used as a load lock was developed which had a capacity of six positions and could expose one position (three samples) at a time to the magnetron. The gas pressure was adjusted without turning off the magnetron.

As shown in Fig. 1, the deposited films were then patterned using a positivetone photoresist into a TES structure of $(6 \times 8) \mathrm{mm}^{2}$ and the uncovered $\mathrm{W}$ was wetetched. Then, a 1-mm-wide stripe was structured using a negative-tone photoresist and a 500-nm Au layer was deposited in a lift-off process for the purpose of thermal anchoring. A similar process was done to deposit two aluminum bias bond pads on both ends. For $T_{c}$ measurements, the samples were mounted on a copper holder and wire-bonded using aluminum wire for the bias current connection and a gold wire for thermal anchoring. The holder was mounted in a dilution refrigerator that has a 
Fig. 1 A schematic of the tested TES (Color figure online)

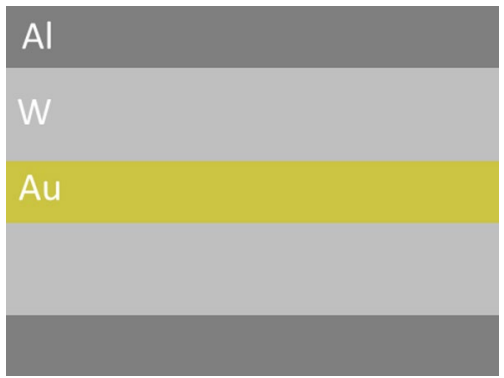

base temperature of $<10 \mathrm{mK}$. The TESs were voltage-biased using a $40 \mathrm{~m} \Omega$ shunt resistor, and their resistance was measured using a SQUID amplifier [19].

\section{Results}

Figure 2 shows typical transition curves of resistance $(R)$ versus temperature $(T)$ of two W-TESs deposited at 0.6 $\mathrm{Pa} \mathrm{Xe}$. Two TESs were patterned on the same substrate to study the homogeneity of the film as well as the possible scatter in the in $T_{c}$ in the same deposition run. The films have a $T_{c} \sim 16 \mathrm{mK}$ and $\Delta T_{c} 0.4 \mathrm{mK}$. The two TESs have a difference in $T_{c}$ of $\sim 0.5 \mathrm{mK}$ which is acceptable for our purpose (for the experiment transition temperatures between 15 and $20 \mathrm{mK}$ are acceptable).

Figure 3 shows the $T_{c}$ of $\mathrm{W}$ films versus the Xe pressure. Despite the still low statistics, it is possible to presume a linear correlation between deposition pressure and $T_{c}$.

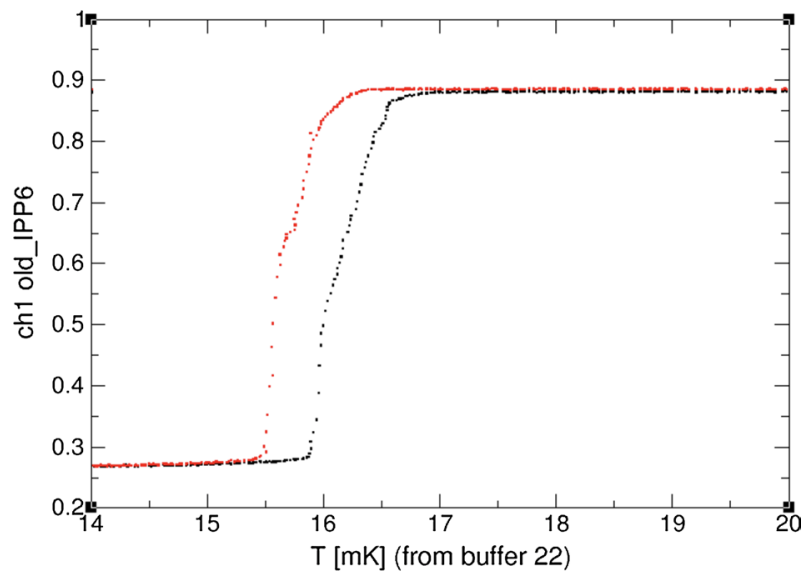

Fig. 2 A comparison between two $8 \mathrm{~mm}^{2}$ TESs structured on the substrate. They were both biased with the same current of $1.0 \mu \mathrm{A}$. A shift of $0.5 \mathrm{mK}$ can be seen (Color figure online) 


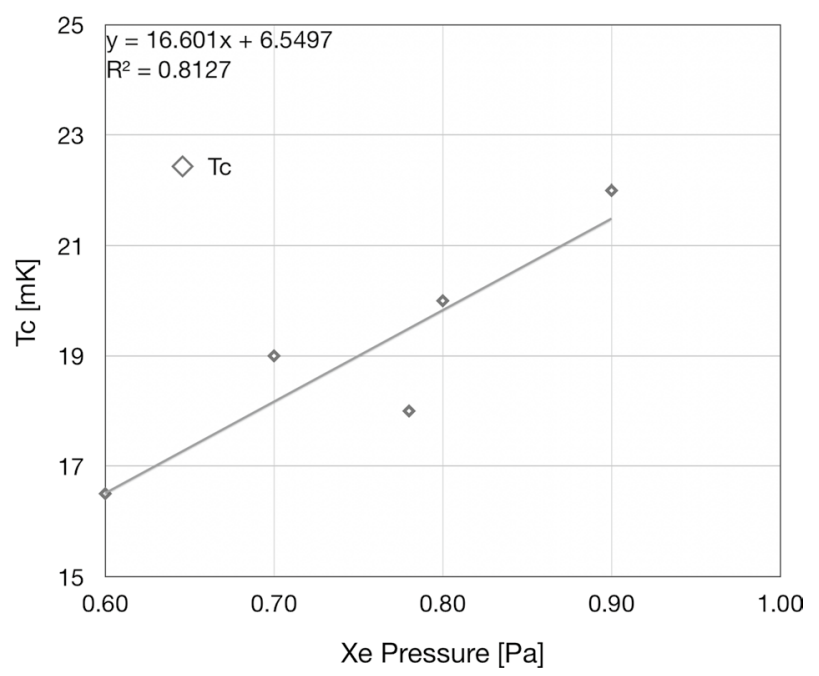

Fig. $3 T_{c}$ of films that were deposited with Xe as a function of sputtering pressure. The pressure was varied in steps of $\sim 0.1 \mathrm{~Pa}$

\section{Discussion}

This work demonstrated that sputtering can produce $\mathrm{W}$ films with properties meeting the requirements for the TES sensors needed by the CRESST experiment. Such method that is potentially scalable to large TES production. Yet further investigation is needed to understand in detail what governs the transition and how to improve the sensitivity of a TES. Further study of the reproducibility is under investigation in a dedicated system at MPP.

So far films have been grown on Si substrates with $\sim 30 \mathrm{~nm}$ thermally grown $\mathrm{SiO}_{2}$. However, the next step is to study films deposited on different substrates, e.g., $\mathrm{CaWO}_{4}$, sapphire etc., following the experimental requirement of having different target nuclei available for the understanding of background sources and for the study of possible dark matter signals.

\section{Conclusion}

Sputtering W films for TES fabrication with Xenon gas showed that a TES with $T_{c}$ down to $15 \mathrm{mK}$ with transition width smaller than $1 \mathrm{mK}$ is achievable. Tuning of $T_{c}$ may be possible by controlling the Xe pressure during the sputter process.

Acknowledgements Open Access funding provided by Projekt DEAL. 
Open Access This article is licensed under a Creative Commons Attribution 4.0 International License, which permits use, sharing, adaptation, distribution and reproduction in any medium or format, as long as you give appropriate credit to the original author(s) and the source, provide a link to the Creative Commons licence, and indicate if changes were made. The images or other third party material in this article are included in the article's Creative Commons licence, unless indicated otherwise in a credit line to the material. If material is not included in the article's Creative Commons licence and your intended use is not permitted by statutory regulation or exceeds the permitted use, you will need to obtain permission directly from the copyright holder. To view a copy of this licence, visit http://creativecommons.org/licen ses/by/4.0/.

\section{References}

1. K.D. Irwin, G.C. Hilton, in Cryogenic Particle Detection (Springer, Berlin, Heidelberg, 2005), pp. 63-150

2. G. Angloher et al., Eur. Phys. J. C 76(1), 25 (2016)

3. G. Angloher et al., Astropart. Phys. 31(4), 270-276 (2009)

4. J. Rayne, M. Mamor, F. Meyer, Phys. Rev. 95(6), 1428 (1954)

5. S. Roth, Diploma Thesis Technische Universität München (2007). http://www.e15.physik.tumue nchen.de. Accessed 28 Jan 2020

6. A.E. Lita et al., IEEE Trans. Appl. Supercond. 15(2), 3528-3531 (2005)

7. E. Lassner, W.D. Schubert, Tungsten: Properties, Chemistry, Technology of the Element, Alloys, and Chemical Compounds (1999)

8. G.F. Hardy, J.K. Hulm, Phys. Rev. 89(4), 884 (1953)

9. R.H. Willens, E. Buehler, Appl. Phys. Lett. 7(1), 25-26 (1965)

10. F.M. Kilbane, P.S. Habig, J. Vac. Sci. Technol. 12(1), 107-109 (1975)

11. Ali E. Aliev, Supercond. Sci. Technol. 21(11), 115022 (2008)

12. B.A. Young et al., Nuclear Instrum. Methods Phys. Res. Sect. A Accel. Spectrom. Detectors Assoc. Equip. 444(1-2), 296-299 (2000)

13. John A. Thornton, D.W. Hoffman, Thin Solid Films 171(1), 5-31 (1989)

14. B. Stritzker, Phys. Rev. Lett. 42(26), 1769 (1979)

15. P.M. Hall, J. Appl. Phys. 36(8), 2471-2475 (1965)

16. F.C. Nix, D. MacNair, Phys. Rev. 61, 1-2 (1942)

17. Jayant S. Shah, M.E. Straumanis, Solid State Commun. 10.1, 159-162 (1972)

18. M. Mamor et al., Appl. Surf. Sci. 91(1-4), 342-346 (1995)

19. G. Wendin, V.S. Shumeiko, arXiv preprint arXiv:cond-mat/0508729 (2005)

Publisher's Note Springer Nature remains neutral with regard to jurisdictional claims in published maps and institutional affiliations. 